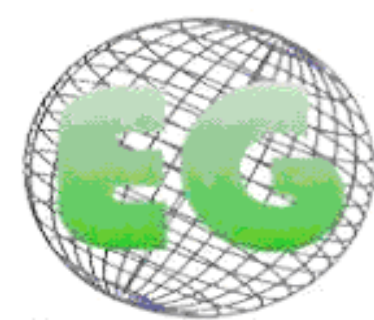

ISSN 1695-6141 $N^{\circ} 22$
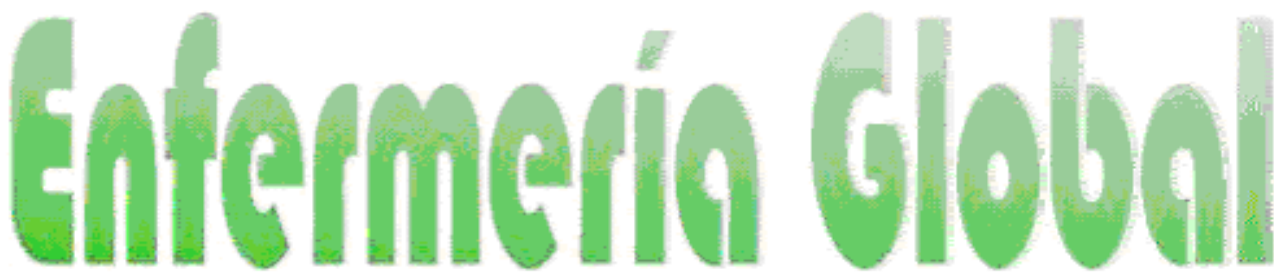

Revista electrónica trimestral de Enfermería

Abril 2011

www.um.es/egloball

\title{
EDUCACIÓN Y ENTRENAMIENTO A PACIENTE Y FAMILIA CON NUTRICIÓN PARENTERAL EN DOMICILIO
}

EDUCATING AND TRAINING THE PATIENT AND FAMILY IN HOME PARENTERAL NUTRITION

\section{*Tejada Domínguez, FJ., "*Ruiz Domínguez, MR. \\ *Zona Básica Villamartín (Cádiz) C.S. Puerto Serrano . **Servicio de Medicina Interna. Hospital Universitario Virgen de Valme (Sevilla). \\ Palabras clave: Nutrición parenteral domiciliaria; Soporte nutricional artificial, Educación sanitaria; Entrenamiento; Aprendizaje. \\ Keywords: Home Parenteral Nutrition; Nutritional Artificial Support; Sanitary Education; TrainingLearning.}

\section{RESUMEN}

La Nutrición Parenteral Domiciliaria consiste en la infusión de nutrientes, a través de una vena, en el propio domicilio del paciente.

Entre sus requisitos, se encuentra el de poder contar con la ayuda, capacitación y colaboración tanto del propio paciente como de la familia y/o cuidador.

Este trabajo representa el programa de educación y entrenamiento dirigido a todos ellos con la finalidad de conseguir que este tipo de soporte se convierta en una modalidad segura y eficaz en cuanto a mantenimiento del estado nutricional y mejora de la calidad de vida.

El contenido se sustenta en una revisión exhaustiva de la literatura científica que abarca estudios, guías de práctica clínica, manuales y otros documentos de consenso avalados por sociedades expertas en nutrición.

Finalizamos concluyendo que este tipo de programas pueden garantizar un adecuado tratamiento nutricional parenteral en el domicilio, favoreciendo la independencia y bienestar del paciente y familia, asegurando una administración segura y evitando ingresos y estancias hospitalarias prolongadas sólo por este motivo.

\section{ABSTRACT}

The Home Parenteral Nutrition consists of the infusion of nutrients, through a vein, carried out at the address of the patient. 
Among its requirements, is that of being able to count on the aid, qualification and collaboration of the patients themselves as well as that of the family and/or caretaker.

This work represents the program of education and training directed to all of them in order to make this type of support a safe and effective modality in terms of maintenance of the nutritional state and improvements in the quality of life.

The content is supported by an exhaustive revision of the scientific Literature that includes studies, guides of clinical practice, manuals and other documents of consensus guaranteed by expert societies in nutrition.

We conclude that these programs can for this reason alone guarantee a suitable parenteral nutritional treatment in the home, so favouring the independence and well-being of the patient and family and assuring a safe administration and avoiding entries and prolonged hospital stays.

\section{INTRODUCCIÓN}

La nutrición artificial en el domicilio $(N A D)$ ha cobrado un importante interés en los últimos años, convirtiéndose en una práctica habitual dentro del soporte nutricional. Esta modalidad surge en respuesta a hospitalizaciones prolongadas de pacientes que, generalmente, presentan una estabilidad clínica y/o evolución satisfactoria, e incluso en los que se ha resuelto el problema patológico y siguen sin poder alimentarse de la forma habitual necesitando por ello permanecer hospitalizados; también aquellos que, por el contrario, mantienen una evolución tórpida y crónica de su patología base con reagudizaciones susceptibles de manejo y seguimiento en su propio domicilio y que su única forma de alimentación es a través de una de las modalidades terapéuticas de soporte nutricional artificial (1).

Una de esas modalidades es la Nutrición Parenteral (NP) y ha supuesto un gran desarrollo en las últimas décadas. El progreso en los sistemas sanitarios con equipos de soporte nutricional muy especializados, la nueva industria farmacéutica (que ha desarrollado a gran velocidad mejores fórmulas, nuevos sistemas, catéteres y bombas de infusión), además de, una correcta programación, planificación y coordinación por parte de los sistemas sanitarios, obteniendo como resultado una mejor capacidad de seguimiento de los pacientes en el domicilio han contribuido a que el entorno domiciliario sea un lugar con plenas garantías para que los pacientes puedan recibir el tratamiento que necesitan sin necesidad de aislarlos de su ambiente social y de asumir innecesariamente largas y costosas estancias hospitalarias.

\section{Definición}

La Nutrición Parenteral consiste en la administración de los nutrientes a través de una vía venosa (generalmente una vía central) en aquellos pacientes incapaces de mantener un estado nutricional adecuado mediante la alimentación oral o la nutrición enteral. En general, se trata de pacientes con fallo intestinal de diferentes etiologías, que impide la absorción de los nutrientes necesarios para cubrir los requerimientos nutricionales; sin este tratamiento, los pacientes verían muy mermadas sus posibilidades de supervivencia (3).

Específicamente se conoce como Nutrición Parenteral Domiciliaria (NPD) al soporte nutricional artificial que se administra en el propio domicilio del paciente utilizando una vía de acceso permanente e indicada de forma transitoria (duración corta, de al menos 1 mes) o indefinida (para toda la vida), o en espera de una posible solución. Entre sus ventajas destacan menor número de complicaciones nosocomiales y posibilidad de adaptación del 
paciente a su medio socio familiar, mayor comodidad para la familia, menor coste económico para el sistema sanitario y reducción de estancias hospitalarias entre otras.

\section{Indicaciones}

Las indicaciones son exactamente las mismas que en el hospital, es decir, enfermos que sean incapaces de recibir adecuadamente por vía gastrointestinal los nutrientes necesarios mediante la dieta oral o nutrición enteral y que pueden ser correctamente atendidos fuera del hospital.

Esta modalidad se utiliza sobre todo en aquellos pacientes con indicación de soporte nutricional intravenoso prolongado en el tiempo, sea de forma temporal o definitiva, siendo requisito imprescindible que la situación clínica del paciente permita su atención y seguimiento en domicilio. El tratamiento debe ir dirigido a conseguir unos objetivos claramente definidos y, tanto el ambiente domiciliario como la situación física y psicológica del paciente, familia y/o cuidador, deben ser adecuados para que este tratamiento domiciliario se pueda llevar a cabo.

\section{Contraindicaciones}

La NPD está contraindicada cuando exista otro procedimiento y vía para nutrir al paciente y cuando no le aporte ningún beneficio en cuanto a prolongación de expectativa de vida y calidad de la misma (p. ej. índice de Karfnosky superior a 50). Ha de suspenderse cuando, una vez iniciada y mantenida, se den estas mismas circunstancias (6).

\section{Objetivos Básicos en NPD}

- Evitar o corregir la desnutrición cuando la vía enteral no es adecuada o posible.

- Evitar prolongamientos de la estancia hospitalaria y las posibles complicaciones derivadas del ingreso (p. ej. infecciones nosocomiales...).

- Reducir costes sanitarios.

- Reintegrar al paciente a su entorno socio-familiar.

- Permitir al resto de componentes de la familia retornar a su situación de normalidad en la medida de lo posible (laboral y social habitual).

\section{Condiciones Determinantes}

Las peculiaridades específicas del soporte nutricional obligan a tener en cuentar una serie de consideraciones precisas que ayudan a seleccionar a los pacientes candidatos. Esta modalidad terapéutica exige unos mínimos necesarios para que su administración sea segura y eficaz. Precisa por tanto que un equipo compuesto por médico, enfermera, nutricionista y farmacéutico sea el encargado de dirigir el tratamiento, realizándose una correcta selección del paciente y confeccionando un plan de actuación que incluya desde los objetivos nutricionales a corto y largo plazo, hasta un programa de formación y aprendizaje orientado al paciente, familia y/o cuidador.

Antes de enviar a su domicilio a un paciente en esta situación, hay que tener en cuenta una serie de factores centrados en una indicación correcta, sistema sanitario capacitado para realizarla y el paciente con entorno familiar capacitado para recibirla [Ver tabla I] (6-7). El 
uso de NPD se debe reevaluar a lo largo del tiempo en función del tratamiento y la evolución de las enfermedades que la han indicado.

Tabla I. Condiciones para la indicación de NPD

\section{Referidas al paciente}

* Imposibilidad de nutrición adecuada y suficiente por vía oral-enteral.

Situación clínica y emocional que permita su tratamiento a domicilio.

Deseo y voluntad del paciente de irse a casa.

Tolerancia a la pauta nutricional.

Expectativa de vida suficiente ( $\geq 3$ meses).

Aceptación del tto. Situación clínica, cognitiva y emocional que asegure el cumplimiento.

Tolerancia demostrada a la NP en los casos de inicio hospitalario.

\section{Referidas al sistema sanitario}

* Disponibilidad de un equipo multidisciplinar de soporte nutricional con experiencia y capacidad de prescripción, seguimiento y asistencia en NPD.

* Disponibilidad de una red eficaz de suministro de la fórmula, el material y el equipamiento.

* Colaboración del equipo continuada y apoyo en todos los cuidados requeridos en casa.

* Elaboración de un plan de actuación concreto que incluya:

- Objetivos nutricionales a corto y largo plazo.

- Programa de formación del paciente, familia o cuidador en la técnica.

- Prescripción de los nutrientes necesarios.

- Vías y pautas de administración adecuadas al ambiente domiciliario.

- Planes de seguimiento y monitorización clínica y de laboratorio del paciente.

\section{Referidas al entorno socio-familiar}

Posibilidad de traslado a domicilio.

Capacidad y motivación para asumir el tratamiento.

Capacidad de algún familiar o allegado para adquirir la formación suficiente.

Ambiente domiciliario con un mínimo de condiciones higiénicas.

Este sistema familiar y de domicilio, puede ser sustituido por algún otro sistema de cuidados prolongados, no hospitalarios (ej. residencias).

\section{MATERIAL Y MÉTODO}

Con el fin de sustentar el contenido educativo y de formación en todo lo relacionado a la NP en el ámbito domiciliario, se ha revisado la extensa literatura existente actualmente. El proceso de búsqueda tuvo que ampliarse cuando se objetivó la escasez de estudios prospectivos aleatorizados con suficiente número de casos para obtener los mejores niveles de evidencia, además de, la diversa variabilidad de la práctica clínica.

No se ha encontrado más y mejor literatura que la que contienen los distintos manuales de nutrición artificial domiciliaria y ambulatoria (NADYA), los documentos y manuales de consenso de la sociedad española de nutrición parenteral y enteral (SENPE), la guía de 
práctica clínica de nutrición parenteral domiciliaria y las recomendaciones del CDC (Center for Disease Control and Prevention) [Véase Anexo] para la reducción de las infecciones asociadas al cateterismo intravascular (17).

Se eligieron los criterios de la Agency for Healthcare Research and Quality para catalogar la evidencia científica disponible según se explican en la Tabla II y la idoneidad de las recomendaciones según la calidad de la investigación. En la Tabla III se definen los distintos grados de recomendación.

Tabla II. Clasificación de la $A H R Q$

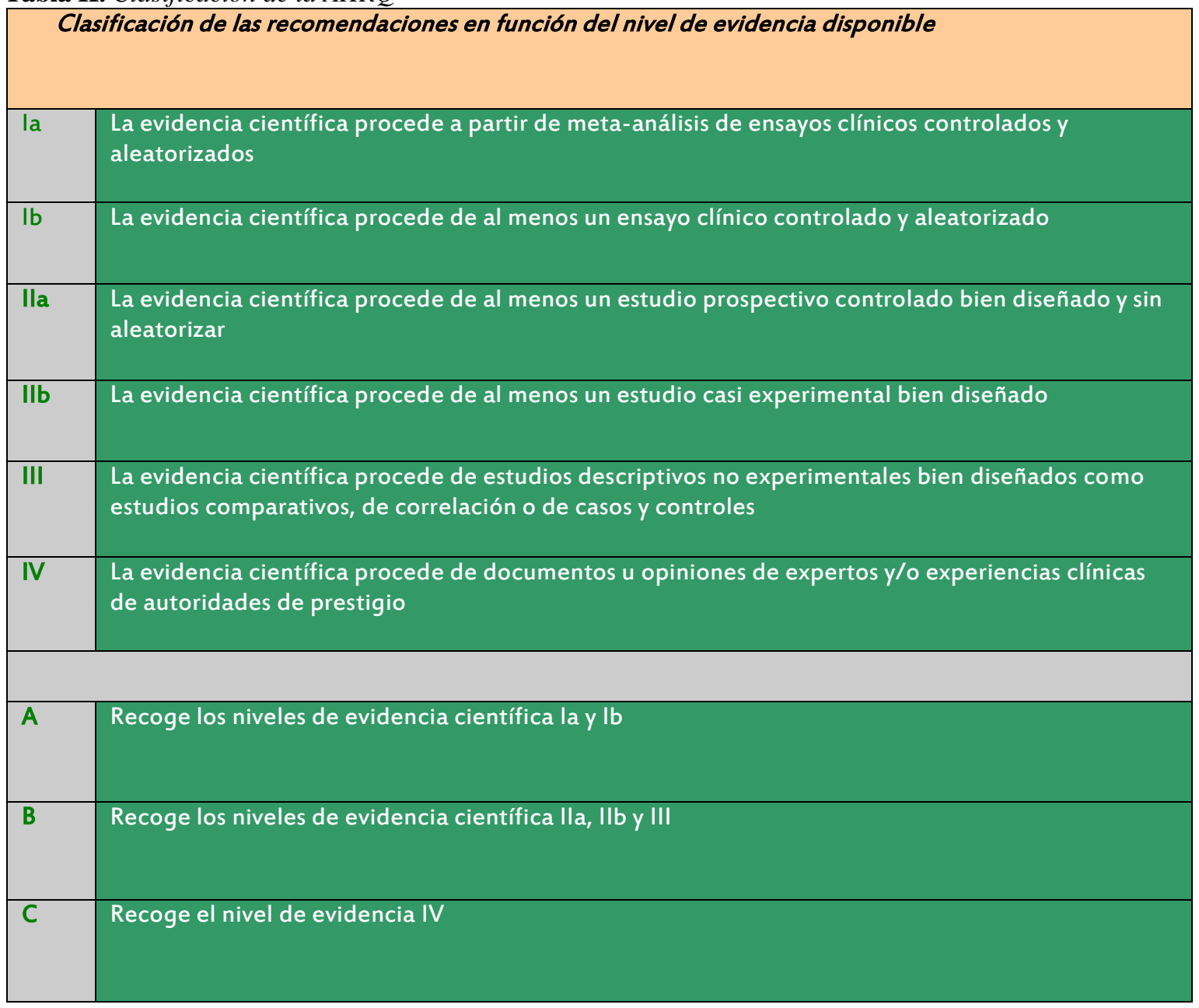

Tabla III. Definición del grado de recomendación

\begin{tabular}{|c|c|}
\hline & Grados de Recomendación \\
\hline $\begin{array}{l}\text { A } \\
\text { B } \\
\text { C }\end{array}$ & $\begin{array}{l}\text { Existe buena evidencia en base a la investigación para apoyar la recomendación } \\
\text { Existe moderada evidencia en base a la investigación para apoyar la recomendación } \\
\text { La recomendación está basada en la opinión de expertos o en un panel de consenso }\end{array}$ \\
\hline
\end{tabular}

\section{EXPOSICIÓN DEL PROGRAMA}

Dado que el objetivo del programa educativo es lograr que el paciente, familia y/o cuidador adquieran los conocimientos y desarrollen las habilidades necesarias para realizar en el hospital todos los cuidados que requiere la administración de NPD, cualquier factor 
determinante para que pueda llevarse a cabo una NPD no resulta eficaz si no se tiene en cuenta el momento, una cuidadosa información y enseñanza de los mismos.

Básicamente, la labor de enseñanza la llevará a cabo el personal de enfermería que, debidamente entrenado a su vez, debe ser el más capacitado para transmitir toda la información necesaria.

La educación debe ser completa pero sencilla y comprensible incluyendo temas teóricos y prácticos distribuidos en varias sesiones de duración limitada y se prolongará hasta confirmar la plena autonomía [Ver Tabla IV]. Será escalonada, progresiva y ajustada a la capacidad de comprensión de la persona, evitando el empleo de terminologías técnicas y comprobando a través de una evaluación teórico-práctica los conocimientos adquiridos respecto a cada tema tratado antes de pasar al siguiente y al finalizar el proceso de educación, momento en el que el paciente o sus cuidadores deber ser capaces de realizar la técnica con total independencia y seguridad (12-13).

\begin{tabular}{|c|c|}
\hline Objetivos y finalidad de la $N P D$ & $\begin{array}{l}\text { - Concepto de } N P \text { y su utilidad. } \\
\text { - Características y composición. }\end{array}$ \\
\hline Características de la vía de acceso venoso & $\begin{array}{l}\text { - } \quad \text { Tipo de catéter. } \\
\text { - } \quad \text { Técnica de colocación. } \\
\text { - Trayecto que recorre. }\end{array}$ \\
\hline Cuidados de la vía de acceso & $\begin{array}{l}\text { - Riesgos y causas de contaminación más frecuentes. } \\
\text { - Medidas para evitarlo. }\end{array}$ \\
\hline $\begin{array}{l}\text { Conocimiento del material, su uso, } \\
\text { manipulación y conservación }\end{array}$ & $\begin{array}{l}\text { - } \quad \text { Elección idónea de la superficie de trabajo. } \\
\text { - } \quad \text { Creación del campo estéril. } \\
\text { - } \quad \text { Formas de cargar jeringuillas. } \\
\text { - } \quad \text { Utilización de tijeras y pinzas sin dañar el material. } \\
\text { - } \quad \text { Técnica de purgado del equipo. } \\
\text { - } \quad \text { Colocación de filtros antipartículas. } \\
\text { - } \quad \text { Manejo de la bomba de infusión, limpieza y } \\
\text { mantenimiento. } \\
\text { Métodos alternativos de infusión: controladores de } \\
\text { flujo. }\end{array}$ \\
\hline $\begin{array}{l}\text { Conocimiento de las normas de higiene } \\
\text { general y personal, condiciones ambientales } \\
\text { adecuadas }\end{array}$ & $\begin{array}{l}\text { - } \quad \text { Lavado de manos correcto. } \\
\text { - } \quad \text { Colocación correcta de guantes. } \\
\text { - } \quad \text { Condiciones de desinfección. } \\
\text { trabajo donde se van a realizar los preparativos y la } \\
\text { administración de la } N P \text {. }\end{array}$ \\
\hline $\begin{array}{l}\text { Técnica de inicio, mantenimiento y retirada de } \\
\text { la } N P\end{array}$ & $\begin{array}{l}\text { - Cíclica o continua. } \\
\text { - Según tipo de catéter: } \\
\text { Reservorio s.c: delimitación de la zona } \\
\text { cutánea, limpieza de la zona, manejo de la } \\
\text { aguja especial, cierre y apertura de la pinza, } \\
\text { lavado y sellado del catéter. } \\
\text { Catéter externo: secuencia de cierre y } \\
\text { apertura de la pinza para evitar entrada de aire, } \\
\text { conexión y desconexión catéter nutrilínea, } \\
\text { lavado y sellado, limpieza y protección de la } \\
\text { conexión. } \\
\text { - Conexión nutrilínea a bolsa de } N P \text { sin dañar ni producir } \\
\text { perforaciones, manera de purgarla, protección hasta que } \\
\text { se acople al catéter. }\end{array}$ \\
\hline
\end{tabular}




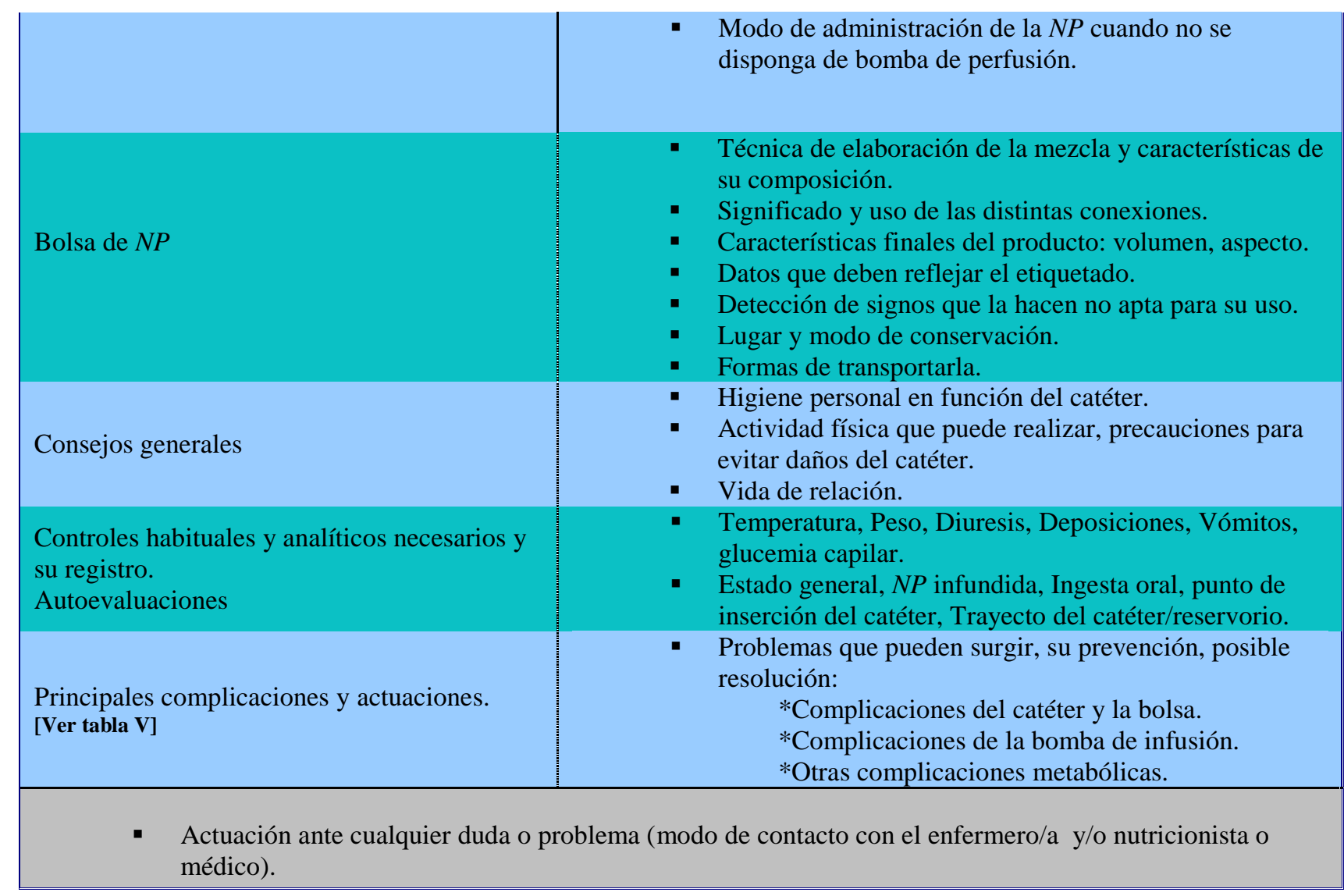

En el proceso de enseñanza resulta conveniente identificar las responsabilidades de instrucción seleccionando por ejemplo de entre los familiares del paciente aquel o aquellos que por razones de convivencia, capacidad y nivel intelectual sean más apropiados (pareja, tutor, etc.). Aún cuando el paciente sea autosuficiente y esté capacitado para cuidarse solo, puede dejar de serlo en cualquier momento y alguien ha de estar entrenado para suplirle o ayudarle. Esta persona, además de recibir información y enseñanza, se mantendrá en contacto con el personal sanitario especializado de forma periódica para poder detectar incumplimientos u otras situaciones de riesgo.

Esta fase de enseñanza va a ser un momento decisivo ya que la aceptación del tratamiento depende, en gran parte, del conocimiento y las habilidades que el paciente y la familia tengan. Su finalidad es evitar temores derivados de conocimientos deficientes o erróneos, que no generan más que una aversión al tratamiento complicando la buena disposición para el aprendizaje.

Al alta hospitalaria se debe verificar el conocimiento y la comprensión de todos los puntos, tanto del procedimiento como de la detección de signos de alarma o la actuación necesaria en caso de presentarse complicaciones. Éstas pueden surgir en cualquier momento y requerir acciones inmediatas [Ver tabla V]. Es importante explicarle y suministrarle un listado lo más completo posible describiendo los síntomas, las causas que los han podido producir, la actitud a tomar y, sobre todo, la mejor manera de prevenirlas. 
Tabla V. Complicaciones e intervenciones más frecuentes en NPD

\begin{tabular}{|c|c|}
\hline COMPLICACIÓN & INTERVENCIONES \\
\hline $\begin{array}{l}\text { Enrojecimiento, inflamación, } \\
\text { supuración o dolor en el punto de } \\
\text { inserción del catéter }\end{array}$ & $\begin{array}{l}\text { - } \quad \text { Evitar uso de cremas antibióticas o pomadas en punto de punción. } \\
\text { - } \quad \text { Recambio periódico de tapones y equipos de infusión. } \\
\text { - } \quad \text { Sustituir el sistema a la vez que la bolsa. } \\
\text { - } \quad \text { Cura antiséptica y sustitución del apósito estéril. } \\
\text { - } \quad \text { Evitener presión positiva en la jeringa al limpiar el catéter. } \\
\text { - } \quad \text { Contactar con equipo. }\end{array}$ \\
\hline Rotura del catéter & $\begin{array}{l}\text { - } \quad \text { Manejar con cuidado. } \\
\text { - } \quad \text { Evitar presiones excesivas al inyectar líquidos con jeringa. } \\
\text { - } \quad \text { Clamparlo por la parte más proximal al punto de inserción. } \\
\text { - } \quad \text { Contactar o acudir al centro sanitario. }\end{array}$ \\
\hline Obstrucción del catéter & $\begin{array}{l}\text { - } \quad \text { Detener la perfusión. } \\
\text { - } \quad \text { Contactar. } \\
\text { - } \quad \text { Pigilar hipoglucemias posibles mientras tanto. } \\
\text { finalizar } N P \text { cíclica (8-16 horas). Irrigar con suero fisiológico. Evitar acodamientos } \\
\text { y mezclas de fármacos junto a la } N P \text {. }\end{array}$ \\
\hline Sangre en el catéter & $\begin{array}{ll}\text { - } & \text { Asegurar las conexiones. } \\
\text { - } & \text { Verificar que el catéter no está roto. } \\
\text { - } & \text { Lavar con suero salino. } \\
\end{array}$ \\
\hline Fallos en la bomba de perfusión & $\begin{array}{l}\text { - Realizar mantenimiento y verificación correcta: } \\
\text { *Comprobar purgas del sistema. } \\
\text { *Evitar acodamientos del sistema y catéter. } \\
\text { *Mantener el sensor de gotas limpio y bien colocado. } \\
\text { *Mantener catéter permeable. } \\
\text { *Verificar conexiones a corriente eléctrica y batería con suficiente } \\
\quad \text { cobertura ante cortes del suministro. } \\
\text { - Comprobar con revisiones periódicas su funcionamiento. } \\
\text { - } \quad \text { Utilizar dosificador de flujo alternativo si bomba inutilizada. } \\
\text { Ponerse en contacto con casa comercial o equipo sanitario. }\end{array}$ \\
\hline Fiebre & $\begin{array}{l}\text { - } \quad \text { Contactar con equipo sanitario o servicio de urgencias. } \\
\text { - } \quad \text { Descartar causas que la justifiquen (catarro, infección urinaria...) } \\
\text { - } \quad \text { Inspeccionar las soluciones para detectar turbideces u otras anomalías. } \\
\text { - Utilizar técnica de manipulaciones lo más aséptica posible. }\end{array}$ \\
\hline Hiperglucemia & $\begin{array}{l}\text { - } \quad \text { Detección de niveles de glucosa en sangre y orina. } \\
\text { - } \quad \text { Mantener la velocidad de perfusión prescrita. } \\
\text { - } \quad \text { Contactar. }\end{array}$ \\
\hline Hipoglucemia & $\begin{array}{l}\text { - Beber vaso de agua azucarada o zumo, chupar } 2 \text { cucharadas de azúcar. } \\
\text { - } \quad \text { Santener la velocidad a ritmo constante. } \\
\text { - de perfusión decreciente en la última hora de administración. } \\
\text { - Contactar. }\end{array}$ \\
\hline $\begin{array}{l}\text { Alteración brusca peso, diuresis o } \\
\text { sensación de sed }\end{array}$ & $\begin{array}{l}\text { - } \quad \text { Contactar con equipo responsable. } \\
\text { - } \quad \text { Hacer análisis glucosa en sangre. }\end{array}$ \\
\hline
\end{tabular}

Se elaborarán los planes de seguimiento y de monitorización clínica y de laboratorio del paciente y se organizará el suministro periódico de material y productos para poder llevar a cabo correctamente este tratamiento (el personal de área de nutrición del hospital va a ser el responsable de la preparación de las bolsas de NPD). Es importante también que, en previsión de algunos problemas, el paciente disponga en su domicilio del material elemental que le permita resolver, al menos temporalmente, las situaciones de emergencia, como son una bolsa de NP de repuesto o envases de suero. También es importante incluir información 
de las características de todo el material que va a precisar, dónde se lo van a suministrar y el modo de contactar con la estructura sanitaria de apoyo en caso de necesitarlo (13).

\section{DISCUSIÓN}

Aunque en nuestro país según se especifica en el Real Decreto 1030/2006, de 15 de septiembre, la inclusión de la NPD en la cartera de prestaciones del Sistema Nacional de Salud no está desarrollada la normativa que especifique el cómo debe llevarse a cabo esta prestación.

Por ello, sin una legislación sobre su uso que incluya, además de la financiación su seguimiento, a través de guías de actuación y sistemas de evaluación, información y acreditación de centros que permitan obtener una información más veraz del asunto unificando y especificando los sistemas de información y registro, es difícil que esta modalidad terapéutica se potencie y se lleve a cabo correctamente.

\section{CONCLUSIONES Y COMENTARIOS}

La nutrición parenteral supone un nuevo enfoque en el cuidado domiciliario que permite, además de garantizar un correcto tratamiento nutricional favoreciendo la autonomía y bienestar del paciente y su familia, disminuir la morbilidad y el coste económico que soporta la hospitalización únicamente por este motivo.

Por y a pesar de ello, deberíamos tener en cuenta las siguientes consideraciones:

1. La existencia de canales de comunicación ágiles y de fácil acceso establecidos de forma multidireccional entre el equipo hospitalario, atención primaria, hospitalización domiciliaria y familia podrían minimizar los problemas relacionados con este tipo de nutrición y facilitar la resolución de los mismos.

2. Como toda actividad emergente y en desarrollo, no debería considerarse como algo estático, sino que tendría que ir adaptándose a los continuos avances de tal forma que los tratamientos se adecuaran a los conocimientos que en cada momento se dispusiesen sobre esta materia.

\section{BIBLIOGRAFÍA}

1. Rico Hernández $M^{a}$.A. Armero Fuster M, Díaz Gómez J, Calvo Viñuela I. Educación y Entrenamiento de los Pacientes con Nutrición Artificial Domiciliaria. En: Calvo Buzos SC, Coordinadora. Manual de Nutrición Artificial Domiciliaria. $1^{a}$ Edición. Madrid: Uned; 2008. p. 484-517.

2. Gómez Candela C, Rosado Iglesias C, De Cos Blanco Al, Castillo Rabaneda R, Mateo Lobo R. Nutrición Artificial Domiciliaria en el Paciente Oncológico. En: Gómez Candela C, Editora. Soporte Nutricional en el Paciente Oncológico. 2 $2^{\underline{a}}$ Edición. Madrid: SEOM; 2004. p. 175-82.

3. García Luna P.P, García Arcal J.C, Navarro Rodríguez A. Seguimiento y 
Monitorización de los Pacientes. En: Celaya Pérez S, Coordinador. Manual de Nutrición Artificial Domiciliaria y Ambulatoria. Procedimientos Educativos y Terapéuticos. 1 ${ }^{a}$ Edición. Zaragoza: Ebrolibro; 2000. p. 125-40.

4. Wanden Berghe, C, Sanz Valero J, Jiménez M, Hernández M. [Grupo de Nutrición de la Federación Española de Hospitalización a Domicilio. «Malnutrition of HomeBased Hospital Patients». Nutr. Hosp. 2006; 21: 22-5.

5. Martínez M.A, Arbones M.J, Bellido D. Nutrición Artificial Domiciliaria: Endocrinol. Nutr. 2004; 51(4):173-8.

6. Guía de Práctica Clínica de Nutrición Parenteral Domiciliaria. Coordinador: Grupo de trabajo de NADYA. Nutr. Hosp. Suplementos. 2009; 2(1):1-36. Disponible en: www.nutriciónhospitalaria.com

7. Manual de Formación al Paciente en Nutrición Parenteral Domiciliaria. Grupo $\begin{array}{llllll}\text { NADYA } & \text { (citado } & 19 & \text { Junio } & \text { 2010) }\end{array}$ http://www.senpe.com/publicaciones/manuales.htm

8. Álvarez Hernández J. Fundamentos Prácticos de la Nutrición Artificial Domiciliaria: Endocrinol. Nutr. 2008; 55(8): 357-66.

9. De Cos Blanco A.I, Gómez Candela C. Recomendaciones para la Práctica de la Nutrición Artificial Domiciliaria y Ambulatoria (N.A.D.Y.A). En: Grupo NADYA (Eds.) Manual de Nutrición Artificial Domiciliaria y Ambulatoria. Zaragoza: Ebrolibro; 2000 189-203.

10.Gomis Muñoz P, et al. Documento de Consenso SENPE/SEGHNP/SEFH sobre Nutrición Parenteral Pediátrica. Nutr. Hosp. 2007; 22:710-19.

11.Steiger E, and the HPEN Working Group. Consensus Statements Regarding Optimal Management of Home Parenteral Nutrition Access. JPEN 2006; 30: 94-5.

12. Rodríguez T. Proceso de Educación del Paciente/Familiar en Programa de Nutrición Parenteral Domiciliaria. Farmacéutico Hospitales 2006; 6:319-25.

13. Burgos R, Planas M. Organización de la Nutrición Parenteral Domiciliaria. Endocrinol. Nutr.2004; 51(4): 179-82.

14. Jiménez Sanz M, Ordóñez González J. Complicaciones Asociadas a la Nutrición Parenteral: Prevención y Tratamiento. Monitorización del Paciente con Nutrición Parenteral. Especificaciones para su Administración Domiciliaria. En: Planas M, Coordinadora. Conceptos Prácticos en Nutrición Enteral y Parenteral. 1aㅡ Edición. Barcelona: Mayo Ediciones; 2008. p. 155-180.

15. Cuerda C, Parón L. Complicaciones Infecciosas en Nutrición Parenteral Domiciliaria. El Farmacéutico Hospitales 2006; 176:30-9.

16. Scolapio JS. A Review of the Trends in The Use of Enteral and Parenteral Nutrition Support. J Clin Gastroenterol 2004; 38:403-7.

17. Guidelines for the Prevention of Intravascular Catéter-Related Infecctions 2002 CDC-USA (Versión en Castellano: Guía de Prevención de Infecciones Relacionadas con Catéteres Intravasculares 2003/04). Gráficas Digarza S.L. Málaga. 2003. 
18. Pomp A, Caldwell MD, Feitelson M. Seldinger Technique for Central Venous Catheter Insertions, a Prospective Study of 200 Cases. Clin. Nutr. 1987; 6 (suppl.):103.

19. Pemberton LB, Lyman b, Lander V, Covinsky J. Sepsis fron Triple-vs Single-Lumen Catheters During Total Parenteral Nutrition in Surgical or Critically ill Patients. Arch Surg 1986; 121: 591-4.

20. Pittet D, Hugonnet S, Harbarth S, Mourouga P, Sauvan V, Touveneau S y cols. Effectiveness of a Hospital-wide Programme to Improve Compliance with Hand Hygiene. Infection Control Programme. Lancet 2000; 356: 1307-12. Erratum in: Lancet 2000;356:2196.

21.Pironi, L. Cost-Benefit of Home Artificial Nutrition Regulation. Clin. Nutr. 2001; 20:101-102.

22. RD 1030/2006, de 15 de Septiembre, por el que se establece la Cartera de Servicios Comunes del Sistema Nacional de Salud y el Procedimiento para su Actualización. Boletín Oficial del Estado, ㄲo 222, (16-09-2006). 